\title{
SHOX haploinsufficiency presenting with isolated short long bones in the second and third trimester
}

\author{
Shwetha Ramachandrappa ${ }^{1} \cdot$ Abhijit Kulkarni $^{1} \cdot$ Hina Gandhi ${ }^{2} \cdot$ Cheryl Ellis $^{3} \cdot$ Renata Hutt $^{4} \cdot$ Lesley Roberts $^{4}$. \\ Rosol Hamid ${ }^{5}$. Aris Papageorghiou ${ }^{6}$. Sahar Mansour ${ }^{1}$
}

Received: 11 June 2017 / Revised: 16 October 2017 / Accepted: 24 October 2017 / Published online: 12 January 2018

(c) European Society of Human Genetics 2018

\begin{abstract}
Haploinsufficiency of the transcription factor short stature homeobox (SHOX) manifests as a spectrum of clinical phenotypes, ranging from disproportionate short stature and Madelung deformity to isolated short stature. Here, we describe five infants with molecularly confirmed diagnoses of SHOX haploinsufficiency who presented in utero with short long bones during routine antenatal scanning from as early as 19 weeks gestation. Other foetal growth parameters were normal. The molecular basis of SHOX haploinsufficiency was distinct in each case. In four cases, SHOX haploinsufficiency was inherited from a previously undiagnosed parent. In our de novo case, SHOX haploinsufficiency reflected the formation of a derivative sex chromosome during paternal meiosis. Final adult height in the SHOX-deficient parents ranged from -1.9 to -1.2 SDS. All affected parents had disproportionately short limbs and two affected mothers had bilateral Madelung deformity. To our knowledge, SHOX haploinsufficiency has not previously been reported to present in utero. Our experience illustrates that SHOX deficiency should form part of the differential diagnosis of foetal short long bones and suggests a low threshold for genetic testing. This should be particularly targeted at, but not limited to, families with a history of features suggestive of SHOX deficiency. Data on the postnatal growth of our index cases is presented which demonstrates that antenatal presentation of SHOX haploinsufficiency is not indicative of severe postnatal growth restriction. Early identification of SHOX deficiency will enable accurate genetic counselling reflecting a good postnatal outcome and facilitate optimal initiation of growth hormone therapy.
\end{abstract}

Sahar Mansour

smansour@sgul.ac.uk

1 South West Thames Regional Genetics Unit, St George's University of London, Cranmer Terrace, London SW17 0RE, UK

2 Department of Obstetrics and Gynaecology, Surrey and Sussex Healthcare NHS Trust, Canada Avenue, Redhill RH1 5RH, UK

3 Department of Obstetrics and Gynaecology, Epsom and St Helier University Hospitals NHS Trust, Wrythe Lane, Carshalton SM5 1AA, UK

4 Department of Obstetrics and Gynaecology, Royal Surrey County Hospital NHS Foundation Trust, Egerton Road, Guildford GU2 7XX, UK

5 Department of Obstetrics and Gynaecology, Croydon Health Services NHS Trust, London Road, Croydon CR7 7YE, UK

6 Fetal Medicine Unit, St George's University of London, Cranmer Terrace, London SW17 ORE, UK

\section{Introduction}

Antenatally detected short long bones in the presence of normal cranial and abdominal growth parameters present a diagnostic and counselling challenge. Constitutional small stature and early placental insufficiency are diagnoses of exclusion, which should only be considered once the possibility of cytogenetic abnormalities and the presence of an underlying skeletal dysplasia have been explored. With the growing availability of prenatal array comparative genomic hybridisation (array CGH) pertinent cytogenetic changes can now be readily detected. Despite the increasing accessibility of high-throughput sequencing technology, accurate antenatal diagnosis of skeletal dysplasias is impeded by their broad genetic and phenotypic heterogeneity [1,2]. Features of skeletal dysplasia such as epiphyseal stippling, bowing of the long bones, aberrant bone mineralisation, and fractures are not always present on antenatal scanning. Furthermore, disproportionate limb development may be a late sign as exemplified by achondroplasia where short long bones are rarely detected before the third trimester $[3,4]$. 
The short stature homeobox (SHOX)-containing gene encodes a paired related homeodomain transcription factor, which plays an essential and dosage-sensitive role in linear growth, and in limb development. In the complete absence of SHOX, patients develop Langer Mesomelic Dysplasia (LMD), a skeletal dysplasia characterised by marked mesomelic and rhizomelic limb shortening resulting in severe disproportionate short stature [5].

SHOX haploinsufficiency results in a range of phenotypes. In its most severe form, SHOX haploinsufficiency causes Leri-Weill Dyschondrosteosis (LWD), which is classically characterised by short stature, mesomelic limb shortening, and an abnormality of the forearms known as Madelung deformity [6, 7]. Madelung deformity describes the bowing of the radius and the dorsal dislocation of the ulna caused by the premature fusion of the anteromedial portion of the distal growth plate of the radius [8]. Although subtle radiographic changes may be detectable from infancy, visible deformity does not typically present until late childhood [9, 10]. LWD has a female preponderance, which has been suggested to reflect the influence of oestrogen on the growth plate [11].

In its mildest form, SHOX haploinsufficiency may be difficult to diagnose. The most consistent feature in these patients is short stature, with other clinical features being absent, or difficult to detect. The prevalence of molecular defects in SHOX among this group of patients is not clear as relevant studies have varied considerably with regard to the clinical criteria used to recruit subjects, the molecular testing strategies, which have been employed, and importantly their evaluation of control subjects [12-14]. A study examining the coding region and downstream enhancer elements of SHOX in a cohort of French children with short stature (height of -2 SDS or less), suggested that SHOX haploinsufficiency may account for as many as $17 \%$ of cases of isolated short stature [15].

SHOX is encoded in the pseudoautosomal region 1 (PAR1) at the distal end of the $\mathrm{X}$ and $\mathrm{Y}$ chromosomes; a highly repetitive region, which is susceptible to deletion and duplication events. The molecular basis of SHOX haploinsufficiency is diverse comprising partial or whole gene deletions, partial gene duplications, deletions and duplications of upstream or downstream enhancer elements and missense sequence variants [16-20]. Detailed comparison of patients with similar genotypes at the SHOX locus has yet to reveal any clear genotype-phenotype correlations.

Although longitudinal studies have suggested that failure of linear growth in SHOX haploinsufficiency is measureable from an early age, SHOX deficiency is rarely diagnosed before late childhood [9, 10, 21]. Recombinant growth hormone administered by daily subcutaneous injection is a safe and effective treatment for growth failure in SHOX haploinsufficiency [22].
Here, we report five infants with antenatally detected short long bones attributable to SHOX haploinsufficiency. These cases demonstrate that $S H O X$ regulates skeletal development in utero and suggest that SHOX deficiency is an important differential for moderately shortened long bones in the second and third trimesters. Early diagnosis of SHOX deficiency will enable accurate genetic counselling and may facilitate the optimal timing of hormonal treatment.

\section{Subjects and methods}

All patients included in this study were referred to the clinical genetics service following the identification of short long bones on routine antenatal scanning. Phenotypic data were collected by review of medical records, review of imaging, patient contact, and clinical photographs. Each patient has been examined by at least one of the authors. In this report, LWD denotes the presence of relative short stature, mesomelia and Madelung deformity with or without other features of SHOX deficiency. Limb disproportion was characterised as affecting proximal segments (rhizomelic shortening) or intermediate segments (mesomelic shortening) by clinical evaluation of the surface anatomy. Formal assessment of limb proportions by radiograph was not undertaken. Anthropomorphic measures were made using standard equipment.

Genomic DNA was extracted using standard protocols. Screening of SHOX for missense sequence variants, deletions, and duplications, was performed by Sanger sequencing and multiplex ligation-dependent probe amplification (MLPA) analysis. Array CGH data were examined using bluefuse analysis software. Cell preparations and karyotype analysis were done using standard protocols. All variants are publically available at the gene variant database https://data bases.lovd.nl/shared/genes/SHOX (patient IDs 00116800, 00116798, 00116801, 00115467, and 00117188).

\section{Results}

\section{Case 1}

A 19-year-old woman, gravida 3 para 0 , underwent routine ultrasound examination at $20+1$ weeks gestation revealing a discrepancy in the foetal measurements with significant shortening of the limbs (Table 1). No additional structural abnormalities were detected. Uterine artery dopplers demonstrated normal placental blood flow (sum of pulsatility indices for the uterine arteries $<2.5$ ) and first trimester aneuploidy screening predicted that the foetus was unlikely to be affected by trisomy 21 . 








Fig. 1 Pedigrees of the families presented in this study. Individuals who have molecularly confirmed SHOX haploinsufficiency are shaded in black. Individuals with OI type 1 are shaded in grey. Height SDS is shown where available. The arrow in each pedigree indicates the infant in each of our cases presenting in utero with short long bones on routine antenatal scanning 
The patient (II-2 in Family A, Fig. 1) had a dominant family history of mild short stature. On examination, her head circumference was $58.0 \mathrm{~cm}$ (3.3 SDS), her height was $151.5 \mathrm{~cm}(-1.8 \mathrm{SDS})$, her sitting height was $80.0 \mathrm{~cm}(-2.5$ SDS), her arm span was $133.0 \mathrm{~cm}$ (arm span/height ratio, which should approach 1 was 0.88 ), and her lower segment was $74.0 \mathrm{~cm}$ (upper segment/lower segment ratio, which should approach 1 was 1.05). Her pre-pregnancy weight was $59.5 \mathrm{~kg}$ (0.2 SDS). She had a high arched palate, sloping shoulders, and short forearms with bilateral Madelung deformity (panels a and b, Fig 2). Examination of the father was unremarkable; his height was $178.0 \mathrm{~cm}(0.5$ SDS).

Molecular analysis of the SHOX gene in a maternal blood sample by MLPA revealed a heterozygous deletion of exons 1-6 confirming a diagnosis of LWD (Table 1). A female infant was delivered at $38+4$ weeks gestation weighing $2.551 \mathrm{~kg}$ (2-9th centile). On examination at 8 months, her head circumference was $45.0 \mathrm{~cm}\left(50^{\text {th }}\right.$ centile), and her length was $66.0 \mathrm{~cm}$ (9th centile). The infant had mesomelic limb shortening; analysis of the SHOX locus verified that she had inherited her mother's whole gene deletion.

\section{Case 2}

A 21-year-old woman, gravida 2 para 0 , underwent routine ultrasound examination at 20 weeks gestation, which was unremarkable. A further antenatal scan was organised at $29+3$ weeks gestation in view of maternal illness. This examination detected significant shortening of the limbs (Table 1). Placental blood flow was normal and karyotype analysis of an amniotic fluid sample did not detect any cytogenetic abnormalities.

Maternal examination was unremarkable; her height was $165.0 \mathrm{~cm}$ (0.5 SDS). The father (II-2 in Family B, Fig. 1) had a family history of short stature. On examination, his weight was $72.0 \mathrm{~kg}(0.4 \mathrm{SDS})$, his head circumference was $55.5 \mathrm{~cm}$ (0.3 SDS), his height was $162.0 \mathrm{~cm}$ (-1.9 SDS), and his sitting height was $91.0 \mathrm{~cm}(-0.8 \mathrm{SDS})$. He had sloping shoulders with rhizomelic limb shortening and muscular hypertrophy. He did not have a Madelung deformity (panel c, Fig 2).

Molecular analysis of the SHOX gene in the father revealed that he was heterozygous for a missense sequence variant in exon 4 of SHOX (Table 1). This variant resulted in the substitution of a tryptophan residue for a serine residue within the homeobox domain of the protein and has previously been reported as affecting protein function.

A female infant was delivered at $40+6$ weeks gestation weighing $3.115 \mathrm{~kg}$ (25-50th centile). Her birth length was $48.0 \mathrm{~cm}(9-25$ th centile). On examination at 2 months of age, her head circumference was $39.0 \mathrm{~cm}$ (50th centile), her length was $56.0 \mathrm{~cm}$ (25th centile), and her weight was $5.440 \mathrm{~kg}$ (50-75th centile). She had rhizomelic limb shortening (panel d, Fig 2). Sequence analysis of the SHOX gene confirmed that she had inherited the missense sequence variant that had been found in her father.

\section{Case 3}

A 42-year-old woman, gravida 5 para 1, underwent routine ultrasound examination at $19+6$ weeks. Long bone measurements were predominantly in the normal range but with relative shortening of the fibulae and ulnae (Table 1). Specialist input was requested in the pregnancy because the patient had a dominant family history of Osteogenesis Imperfecta (OI) type 1.

Since the patient herself (II-2 in Family C, Fig. 1) had never had any fractures she was reassured that her pregnancy was unlikely to be at risk of being affected by OI type 1. Importantly, there was a dominant family history of short stature, which did not completely segregate with the OI in the family. On examination, her height was $155.0 \mathrm{~cm}$ (-1.2 SDS). She had bilateral Madelung deformity and mesomelic limb shortening. Examination of the father was unremarkable.

A clinical diagnosis of LWD was made; fluorescence insitu hybridisation of the coding and enhancer regions of SHOX as well as Sanger sequencing of the coding exons of SHOX was undertaken, but no clinically significant changes were identified. Antenatal scanning at $25+6$ weeks, $32+1$ weeks, and $36+1$ weeks demonstrated persistent relative shortening of the fibulae and ulnae suggesting that the foetus was likely to have inherited LWD.

A male infant was delivered at 40 weeks gestation. At 17 days of age, he weighed $3.500 \mathrm{~kg}$ (25-50th centile) and his length was $51.5 \mathrm{~cm}$ (25-50th centile). On examination at 15 months of age, his length was $77.5 \mathrm{~cm}$ (25-50th centile) and he had mesomelic shortening of his lower limbs. The advent of MLPA analysis of the SHOX gene confirmed that mother and baby were both heterozygous for a deletion of exons 4-6b corresponding to an intragenic deletion within the SHOX homeodomain (Table 1).

\section{Case 4}

A 33-year-old woman, gravida 2 para 1, underwent routine ultrasound examination at $20+6$ weeks gestation, which demonstrated significant shortening of the limbs (Table 1). No other structural abnormalities were detected and first trimester aneuploidy screening predicted a 1:10,000 risk of the pregnancy being affected by trisomy 21 .

The patient (II-2 in Family D, Fig. 1) had a dominant family history of mild short stature. Her head circumference 
was $55.0 \mathrm{~cm}(0.6 \mathrm{SDS})$ and her height was $154.1 \mathrm{~cm}(-1.3$ SDS). Her pre-pregnancy weight was $63.6 \mathrm{~kg}$ (0.4 SDS). She had short forearms with a prominent lumbar lordosis. Examination of the father was unremarkable; his height was $180.4 \mathrm{~cm}$ (0.8 SDS).

Genetic testing of SHOX in a maternal blood sample was organised based on the patient's family history and clinical examination findings. Sequencing analysis revealed a single base pair deletion in exon 3 of SHOX leading to a frameshift and premature termination of the protein sequence (Table 1).

A male infant was delivered at 42 weeks gestation. Genetic testing confirmed that he had inherited his mother's single base pair deletion in SHOX. On examination at 2 months of age, his head circumference was $38.5 \mathrm{~cm}$ (2-50th centile), his length was $56.4 \mathrm{~cm}$ (9-25th centile), and his weight was $6.320 \mathrm{~kg}$ (75-91st centile). His growth was proportionate. At 5 months of age, his head circumference was $43.8 \mathrm{~cm}$ (50th centile), his length was 63.0 $\mathrm{cm}$ (9th centile), and his weight was $8.290 \mathrm{~kg}$ (75-91st centile). At that stage, his legs were short in comparison with his trunk. His growth was monitored closely and he was started on daily subcutaneous growth hormone therapy at 3 years of age when his height fell to the 2 nd centile. When he was last seen at 4 years of age he had sloping shoulders, a pectus deformity of the chest and short legs. His linear growth had improved in response to growth hormone therapy and his height was on the 25th centile.

\section{Case 5}

A 36-year-old woman, gravida 3 para 1, who had lost a previous pregnancy with trisomy 21 requested chorionic villus sampling at $12+5$ weeks gestation for aneuploidy testing. Quantitative Fluorescence-Polymerase Chain Reaction analysis of chromosomes 13, 18, 21, and X was normal; however, an additional solitary $\mathrm{Y}$ chromosome sequence was unexpectedly amplified prompting further cytogenetic studies. Karyotype analysis followed by array CGH demonstrated an unbalanced translocation of the sex chromosomes. The foetus had a normal X chromosome and a sex chromosome comprised of material from the $\mathrm{X}$ and $\mathrm{Y}$ chromosomes as a result of a de novo translocation between the short arm of the X chromosome and the long arm of the $\mathrm{Y}$ chromosome in paternal meiosis described as 46,X,der $(\mathrm{X}) \mathrm{t}(\mathrm{X} ; \mathrm{Y})(\mathrm{p} 22.3 ; \mathrm{q} 11.22)$. Array $\mathrm{CGH}$ delineated the region of imbalance as being NC_000023.10:g.(?_60726)_ (3253817_3267652)del showing that the foetus was monosomic for a $3.2 \mathrm{Mb}$ region of Xp encompassing PAR1 and extending approximately $600 \mathrm{~kb}$ beyond the PAR1 boundary into the non-PAR1 region. Importantly, the Yq derived material was non-coding and therefore unlikely to have any clinical consequences.
Ultrasound examination at $19+3$ weeks detected significant shortening of the long bones (Table 1). There was no relevant family medical history and examination of both parents (I-1 and I-2 in Family E, Fig. 1) was unremarkable; maternal height was $168.0 \mathrm{~cm}(1.0 \mathrm{SDS})$ and paternal height was $195.0 \mathrm{~cm}$ (3.1 SDS).

The infant was delivered at $41+5$ weeks gestation weighing $3.730 \mathrm{~kg}$ (50-75th centile). Her head circumference at birth was $37.0 \mathrm{~cm}$ (98th centile) and her birth length was $53.0 \mathrm{~cm}$ (91st centile). On examination at 3 weeks of age, her weight was $4.280 \mathrm{~kg}$ (75-91st centile), her head circumference was $38.5 \mathrm{~cm}$ (98th centile), and her length was $52.4 \mathrm{~cm}$ (50th centile). Her limbs were slightly short compared with her trunk; the remainder of her clinical examination, including examination of the external genitalia was unremarkable. When she was last reviewed at 16 months of age her height was on the 50th centile and she was growing proportionately. At that stage, she did not have any significant medical or developmental problems.

\section{Discussion}

SHOX haploinsufficiency caused by deletions, duplications, and missense sequence variants involving the SHOX locus presents with a variable clinical phenotype of which the most consistent feature is short stature [23]. Although failure of linear growth is reported from an early age, in the absence of a family history of SHOX haploinsufficiency the condition is rarely diagnosed before late childhood when additional clinical features may become apparent. It is clear, from published reports that many cases of SHOX haploinsufficiency with a mild phenotype remain undiagnosed. Here, we describe five cases of SHOX haploinsufficiency, which presented prenatally with short long bones on routine antenatal scanning.

Studies of SHOX expression in human embryos have demonstrated SHOX mRNA expression in the undifferentiated mesenchymal tissue of the limb buds from 33 days post conception. The subsequent spatial and temporal pattern of SHOX expression within the developing mesenchyme suggests that it is involved in primordial skeletal formation [24]. A study where a human isoform of SHOX was expressed under the control of a murine COL2A1 promoter and enhancer in a transgenic mouse model, and gene expression was examined by microarray, has suggested that SHOX regulates the levels of several extracellular matrix genes during early limb development [25].

LMD where there is complete absence of functional SHOX protein can present antenatally with severe shortening and possible bowing of the long bones along with absent or hypoplastic ulnae and fibulae [26, 27]. To our 
knowledge, SHOX haploinsufficiency has not previously been reported to present in utero.

The effects of SHOX haploinsufficiency on prenatal growth have been studied by retrospectively examining birth length data from subjects with LWD. The results of these studies have varied depending on the population used for comparison. Compared with population reference values, the mean birth length in patients with LWD was -0.59 SDS, whereas when unaffected siblings were used for comparison, the mean birth length in patients with LWD was reported as -2.14 SDS $[21,28]$. These data suggest that SHOX haploinsufficiency has a significant effect on linear growth in utero.

The detection of short long bones on antenatal scanning should prompt expert ultrasound evaluation of the constituents of the foetal skeleton and the exclusion of concurrent extra-skeletal abnormalities. The presence and pattern of findings can direct further assessment. Isolated short long bones are commonly observed as a manifestation of intra uterine growth retardation (IUGR) and have also been described as a presenting feature of achondroplasia [29]. Here, we expand the differential diagnosis of antenatally detected short long bones to include SHOX haploinsufficiency by describing five cases, which presented prenatally with moderate shortening of the long bones. Four of our cases presented in the second trimester. This is distinct from IUGR, which generally presents in the third trimester, unless there is severe placental insufficiency, which would be reflected in abnormal uterine artery Doppler waveforms and low levels of pregnancy-associated plasma protein A in the first trimester. It also distinguishes SHOX haploinsufficiency from achondroplasia where long bone growth is well characterised as being preserved until 22 weeks gestation [30].

In each of our inherited cases, the diagnosis of SHOX haploinsufficiency was made by targeted genetic analysis of the SHOX locus in a previously undiagnosed parent with clinical features that were suggestive of SHOX deficiency. Affected parents heights' ranged from -1.9 to -1.2 SDS. This is consistent with previous reports suggesting that the mean adult height in SHOX deficiency is variable, and that even within the context of clinical cohorts with their inherent ascertainment bias, many individuals with SHOX deficiency have heights within the normal range [9]. In three cases, mesomelic limb shortening, which is a frequently associated with SHOX haploinsufficiency was seen. In one case, rhizomelic limb shortening, which is less characteristic, but was described among the earliest families with molecularly confirmed SHOX haploinsufficiency, was noted $[6,31]$. The distinct but overlapping clinical characteristics seen in affected parents encapsulates the variable expressivity of SHOX haploinsufficiency. The generous influence of modifying factors on the phenotypic consequences of
SHOX deficiency is also reflected in the postnatal phenotypes observed in our series; case 5 where both parents were of above average height exemplifies that SHOX haploinsufficiency can be well tolerated within a favourable genetic context.

The SHOX gene was originally cloned by comparing the heights of individuals with terminal deletions and interstitial rearrangements of PAR1 to delineate the region, which was critical to linear growth [31-33]. Our de novo case of SHOX haploinsufficiency was haploinsufficient for SHOX due to a translocation between Xp22.3 and Yq11.22 during paternal meiosis resulting in a derivative chromosome, which was monosomic for the distal portion of Xp extending $600 \mathrm{~kb}$ beyond the PAR1 region. Unbalanced translocations between the $\mathrm{X}$ and $\mathrm{Y}$ chromosomes have previously been described in patients with LWD [34, 35]. These reports suggest that if the monosomic region of Xp extends beyond PAR1 male carriers of the derivative chromosome may be more severely affected than their female counterparts due to the contiguous gene deletion of regions, which are unique to the $\mathrm{X}$ chromosome [36-38]. In our patient, the deleted region included Arylsulfatase E, which is known to cause Chondrodysplasia punctata in males. The clinical relevance of the other genes within the deleted region is unclear.

Early diagnosis of SHOX haploinsufficiency in our patients enabled accurate prenatal genetic counselling. In inherited cases, parents were relieved to receive a diagnosis, which their personal experience had informed them had a good prognosis. In our de novo case, the diagnosis was overshadowed by the other consequences of the translocation. Prenatal diagnosis also facilitated optimal timing of any growth hormone treatment. Administration of recombinant human growth hormone by daily subcutaneous injections is a safe and effective therapeutic option for patients with short stature secondary to SHOX deficiency [39-41]. In a prospective multicentre study with longitudinal follow-up, 57\% of patients with SHOX deficiency treated with recombinant human growth hormone attained a final height within the population reference range; similar figures were observed among SHOX-deficient patients treated in routine clinical practice $[22,40]$.

Our experience suggests that SHOX deficiency should form part of the differential diagnosis of generalised or localised moderate shortening of the foetal long bones in the context of normal bone density and mineralisation. We advocate a low threshold for genetic testing particularly where there is a dominant family history of short stature or features suggestive of SHOX deficiency in one of the parents. However, given the variable phenotype and incomplete penetrance of SHOX deficiency, it should be carefully considered in all antenatal presentations of isolated short 
(a)

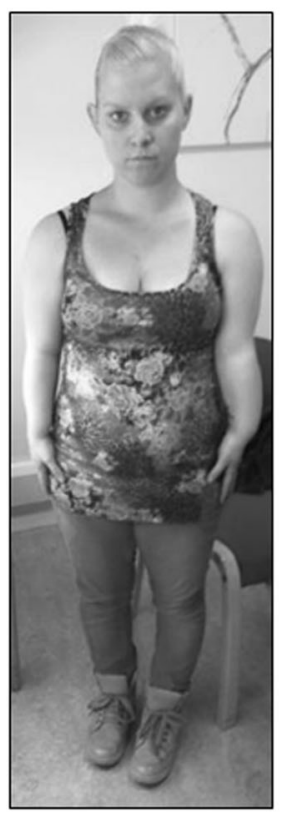

(b)



(c)

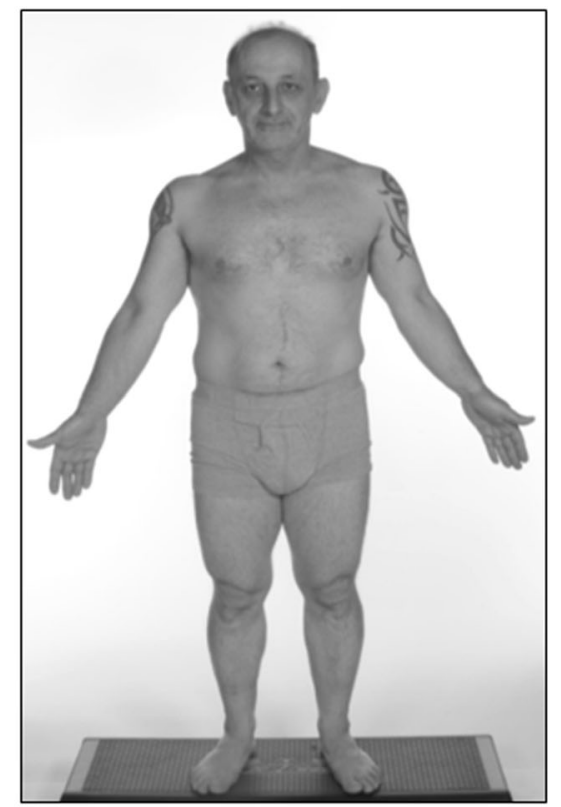

(d)

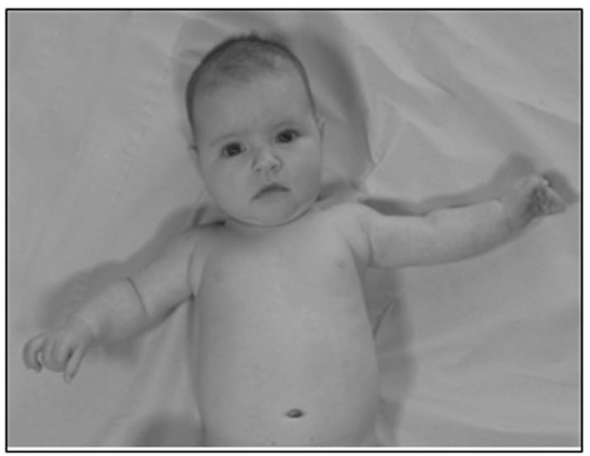

Fig. 2 Clinical photographs of individuals with molecularly confirmed SHOX haploinsufficiency. a, b Affected mother in case 1, II-2 in Family A showing mesomelic upper limb shortening, sloping shoulders and Madelung deformity. c Affected father in case 2,
II-2 in Family B showing rhizomelic limb shortening, sloping shoulders, and muscular hypertrophy. d Affected female infant in case 2, III-2 in Family B at 2 months of age showing rhizomelic limb shortening. long bones. Four of our cases presented in the second trimester suggesting that SHOX deficiency should be given particular consideration in early presentations of disproportionate growth. Whether this proves to be a sensitive indicator of $S H O X$ haploinsufficiency in the prenatal context will require more systematic evaluation. Importantly, in our series antenatal presentation of SHOX haploinsufficiency was not reflective of severe postnatal growth restriction and parents should be reassured as such.

Acknowledgements The families are thanked for their participation in this study.

\section{Compliance with ethical standards}

Conflict of interest The authors declare that they have no conflict of interest.

\section{References}

1. Parilla BV, Leeth EA, Kambich MP, Chilis P, MacGregor SN. Antenatal detection of skeletal dysplasias. J Ultrasound Med. 2003;22:255-61.

2. Doray B, Favre R, Viville B, Langer B, Dreyfus M, Stoll C. Prenatal sonographic diagnosis of skeletal dysplasias. A report of 47 cases. Ann Genet. 2000;43:163-9.
3. Patel MD, Filly RA. Homozygous achondroplasia: US distinction between homozygous, heterozygous, and unaffected fetuses in the second trimester. Radiology. 1995;196:541-5.

4. Kurtz AB, Filly RA, Wapner RJ, et al. In utero analysis of heterozygous achondroplasia: variable time of onset as detected by femur length measurements. J Ultrasound Med. 1986;5:137-40.

5. Zinn AR, Wei F, Zhang L, et al. Complete SHOX deficiency causes Langer mesomelic dysplasia. Am J Med Genet. 2002;110:158-63.

6. Shears DJ, Vassal HJ, Goodman FR, et al. Mutation and deletion of the pseudoautosomal gene SHOX cause Leri-Weill dyschondrosteosis. Nat Genet. 1998;19:70-73.

7. Belin V, Cusin V, Viot G, et al. SHOX mutations in dyschondrosteosis (Leri-Weill syndrome). Nat Genet. 1998;19:67-69.

8. Munns CF, Glass IA, LaBrom R, et al. Histopathological analysis of Leri-Weill dyschondrosteosis: disordered growth plate. Hand Surg. 2001;6:13-23.

9. Ross JL, CJr Scott, Marttila P, et al. Phenotypes associated with SHOX deficiency. J Clin Endocrinol Metab. 2001;86:5674-80.

10. Binder G, Ranke MB, Martin DD. Auxology is a valuable instrument for the clinical diagnosis of SHOX haploinsufficiency in school-age children with unexplained short stature. J Clin Endocrinol Metab. 2003;88:4891-6.

11. Binder G. Short stature due to SHOX deficiency: genotype, phenotype, and therapy. Horm Res Paediatr. 2011;75:81-89.

12. Huber C, Rosilio M, Munnich A, Cormier-Daire V. French SHOX GeNeSIS module. High incidence of SHOX anomalies in individuals with short stature. J Med Genet. 2006;43:735-9.

13. Rappold GA, Fukami M, Niesler B, et al. Deletions of the homeobox gene SHOX (short stature homeobox) are an important cause of growth failure in children with short stature. J Clin Endocrinol Metab. 2002;87:1402-6. 
14. Marstrand-Joergensen MR, Jensen RB, Aksglaede L, Duno M, Juul A. Prevalence of SHOX haploinsufficiency among short statured children. Pediatr Res. 2016;81:335-41.

15. Rosilio M, Huber-Lequesne C, Sapin H, Carel JC, Blum WF, Cormier-Daire V. Genotypes and phenotypes of children with SHOX deficiency in France. J Clin Endocrinol Metab. 2012;97: E1257-E1265.

16. Benito-Sanz S, Aza-Carmona M, Rodriguez-Estevez A, et al. Identification of the first PAR1 deletion encompassing upstream SHOX enhancers in a family with idiopathic short stature. Eur J Hum Genet. 2012;20:125-7.

17. Benito-Sanz S, Royo JL, Barroso E, et al. Identification of the first recurrent PAR1 deletion in Leri-Weill dyschondrosteosis and idiopathic short stature reveals the presence of a novel SHOX enhancer. J Med Genet. 2012;49:442-50.

18. Bunyan DJ, Baffico M, Capone L, et al. Duplications upstream and downstream of SHOX identified as novel causes of Leri-Weill dyschondrosteosis or idiopathic short stature. Am J Med Genet. 2015;170A:949-57.

19. Chen J, Wildhart G, Zhong Z, et al. Enhancer deletions of the SHOX gene as a frequent cause of short stature: the essential role of a $250 \mathrm{~kb}$ downstream regulatory domain. J Med Genet. 2009;46:834-9.

20. Bunyan DJ, Baker KR, Harvey JF, Thomas NS. Diagnostic screening identifies a wide range of mutations involving the SHOX gene, including a common $47.5 \mathrm{~kb}$ deletion $160 \mathrm{~kb}$ downstream with a variable phenotypic effect. Am J Med Genet A. 2013;161A:1329-38.

21. Munns CF, Glass IA, Flanagan S, et al. Familial growth and skeletal features associated with SHOX haploinsufficiency. J Pediatr Endocrinol Metab. 2003;16:987-96.

22. Benabbad I, Rosilio M, Child CJ, et al. Safety outcomes and nearadult height gain of growth hormone-treated children with SHOX deficiency: data from an observational study and a clinical trial. Horm Res Paediatr. 2016;1:42-50.

23. Rappold G, Blum WF, Sharikova EP, et al. Genotypes and phenotypes in children with short stature: clinical indicators of SHOX haploinsufficiency. J Med Genet. 2007;44:306-13.

24. Clement-Jones M, Shiller S, Rao E, et al. The short stature homeobox gene SHOX is involved in skeletal abnormalities in Turner syndrome. Hum Mol Genet. 2000;9:695-702.

25. Beiser KU, Glaser A, Kleinschmidt K, et al. Identification of novel SHOX target genes in the developing limb using a transgenic mouse model. PLoS ONE. 2014;9:e98543.

26. Shears DJ, Guillen-Navarro E, Sempere-Miralles M, Domingo-Jimenez R, Scambler PJ, Winter RM. Pseudodominant inheritance of Langer mesomelic dysplasia caused by a SHOX homeobox missense mutation. Am J Med Genet. 2002;110:153-7.

27. Thomas NS, Maloney V, Bass P, Mulik V, Wellesley D, Castle B. SHOX mutations in a family and a fetus with Langer mesomelic dwarfism. Am J Med Genet A. 2004;128A:179-84.
28. Binder G, Renz A, Martinez A, et al. SHOX haploinsufficiency and Leri-Weill dyschondrosteosis: prevalence and growth failure in relation to mutation, sex, and degree of wrist deformity. J Clin Endocrinol Metab. 2004;89:4403-8.

29. Goncalves L, Jeanty P. Fetal biometry of skeletal dysplasias: a multicentric study. J Ultrasound Med. 1994;13:977-85.

30. Chitty LS, Griffin DR, Meaney C, et al. New aids for the noninvasive prenatal diagnosis of achondroplasia: dysmorphic features, charts of fetal size and molecular confirmation using cell-free fetal DNA in maternal plasma. Ultrasound Obstet Gynecol. 2011;37:283-9.

31. Rao E, Weiss B, Fukami M, et al. Pseudoautosomal deletions encompassing a novel homeobox gene cause growth failure in idiopathic short stature and Turner syndrome. Nat Genet. 1997;16:54-63.

32. Ellison JW, Wardak Z, Young MF, Gehron Robey P, LaigWebster M, Chiong W. PHOG, a candidate gene for involvement in the short stature of Turner syndrome. Hum Mol Genet. 1997;6:1341-7.

33. Ballabio A, Bardoni B, Carrozzo R, et al. Contiguous gene syndromes due to deletions in the distal short arm of the human $\mathrm{X}$ chromosome. Proc Natl Acad Sci USA. 1989;86:10001-5.

34. Calabrese G, Fischetto R, Stuppia L, et al. X/Y translocation in a family with Leri-Weill dyschondrosteosis. Hum Genet. 1999;105: 367-8.

35. Palka G, Stuppia L, Guanciali Franchi P, et al. Short arm rearrangements of sex chromosomes with haploinsufficiency of the SHOX gene are associated with Leri-Weill dyschondrosteosis. Clin Genet. 2000;57:449-53.

36. Boycott KM, Parslow MI, Ross JL, et al. A familial contiguous gene deletion syndrome at Xp22.3 characterized by severe learning disabilities and ADHD. Am J Med Genet A. 2003;122A: 139-47.

37. Seidel J, Schiller S, Kelbova C, et al. Brachytelephalangic dwarfism due to the loss of ARSE and SHOX genes resulting from an X;Y translocation. Clin Genet. 2001;59:115-21.

38. Doherty MJ, Glass IA, Bennett CL, et al. An Xp; Yq translocation causing a novel contiguous gene syndrome in brothers with generalized epilepsy, ichthyosis, and attention deficits. Epilepsia. 2003;44:1529-35.

39. Binder G, Schwarze CP, Ranke MB. Identification of short stature caused by SHOX defects and therapeutic effect of recombinant human growth hormone. J Clin Endocrinol Metab. 2000;85: 245-9.

40. Blum WF, Ross JL, Zimmermann AG, et al. GH treatment to final height produces similar height gains in patients with SHOX deficiency and Turner syndrome: results of a multicenter trial. $\mathrm{J}$ Clin Endocrinol Metab. 2013;98:E1383-E1392.

41. Massart F, Bizzi M, Baggiani A, Miccoli M. Height outcome of the recombinant human growth hormone treatment in patients with SHOX gene haploinsufficiency: a meta-analysis. Pharmacogenomics. 2013;14:607-12. 